\title{
Physics with highly-charged ions in an EBIT
}

\author{
J. R. Crespo López-Urrutia, B. Bapat, B. Feuerstein, D. Fischer, \\ H. Lörch, R. Moshammer, and J. Ullrich \\ Max-Planck-Insitut für Kernphysik, Saupfercheckweg 1, \\ D-69117 Heidelberg, Germany \\ R. DuBois \\ University of Missoury, Rolla, USA \\ Y. Zou \\ Fudan University, Shanhai, PR of China
}

\begin{abstract}
After the commissioning of the Freiburg electron beam ion trap, experiments on dielectronic recombination of the low-lying resonances in He-like $\mathrm{Ar}^{16+}$ have been carried out at high resolution. Forbidden transitions (,,coronal lines") of highly charged argon ions in the optical range have been measured with an accuracy around $1 \mathrm{ppm}$. Ions extracted from FreEBIT have been used to perform collision experiments using the Cold Target Recoil-Ion Momentum Spectroscopy (COLTRIMS) technique.
\end{abstract}

Keywords: ion sources, highly charged ions, spectroscopy, ion-atom collisions, ion-electron collisions, recoil ion momentum spectroscopy

\section{Optical spectroscopy}

The new electron beam ion trap facility FreEBIT was developed to study the physics of highly charged ions with special interest in visible spectroscopy, hyperfine structure studies and collision processes. It was designed as a combination of ion trap and ion source [1] which can deliver beams of highly charged ions to perform kinematically complete collision experiments [2]. Simultaneously the EBIT can be used for spectroscopy studies of HCIs in the x-ray, soft xray, VUV, UV, and visible region. The main interest is to obtain data relevant for plasma diagnostics and astrophysics, as well as fundamental information on the hyperfine structure of hydrogenic ions. The design of the apparatus allows a convenient optical access to the central trap region due to its wide radial magnet bore openings. Two quartz lenses are mounted inside of one of those; these invacuo lenses relay the image of the trap to a plane just outside the quartz vacuum 
window. From there, a set of mirrors and lenses rotate the orientation of the image from horizontal to vertical and project it onto the entrance slit of a grating monochromator. This instrument can reach a dispersion of $0.34 \mathrm{~nm} / \mathrm{mm}$ at the focal plane. Cryogenically cooled CCD detectors with $2000 \times 800$ pixels on a 30 $\mathrm{mm} \times 8 \mathrm{~mm}$ surface are used to detect the incident radiation with very low noise levels. With the two available monochromators, four gratings and two CCD detectors, the energy region from $190 \mathrm{~nm}$ to $850 \mathrm{~nm}$ can be covered with very good resolution and quantum efficiency.

In the first mode of observation, the trap is imaged onto the narrow entrance slit, to approach the resolution limit of the instrument. Narrow spectral lines from the trapped ions are thus obtained. By positioning spectral lamps at the position of the first real image of the trap that appears just outside the quartz window, calibration spectra are recorded before and after each exposure. Afterwards, the grating is slightly rotated. A new exposure with its corresponding calibrations is then recorded. The entire process is repeated as many as thirty times. In this way, the statistical limitations posed by the small number of pixels illuminated across the width of the spectral line are overcome, and the sampling of the line profile is improved by recording several hundred data points for each line profile. Each spectrum is evaluated by fitting individual Gauss functions to the calibration lines (roughly 10 lines on each spectrum) and then using a least-squaresalgorithm to obtain a second degree polynomial for the dispersion function. Since the deviation from the linear dispersion for this type of grating monochromator is small, a quadratic function provides already a very good approximation to the real dispersion. Typical parameters are: At 3.5 pixels FWHM, and a linear dispersion of $0.0086 \mathrm{~nm} / \mathrm{pixel}$, a calibration line has a $3 \cdot 10^{-2} \mathrm{~nm}$ FWHM. The integral number of counts on the line is about 20000 . The centroid of this line can be so determined to better than $1 / 100$ of the line profile when enough data points are recorded and the line profile is well understood. That provided, a $2 \cdot 10^{-4} \mathrm{~nm}$ centroid determination is achieved. The wavelength calibration data are taken from sources where they are given with an accuracy of $10^{-4}$ to $10^{-5} \mathrm{~nm}$. With ten calibration lines spawning the central region of the detector (about $15 \mathrm{~nm}$ ), the standard deviation of the calibration lines from the fitted dispersion curve is $1.6 \cdot 10^{-4}$ $\mathrm{nm}$, agreeing well with the statistical uncertainty of the individual centroid determination. The fitted curve is more accurate than the individual calibration line by a factor roughly proportional to the square root of the number of calibration lines (10) minus the number of degrees of freedom for the fitting function (3). In this case we obtain a numerical result of $6 \cdot 10^{-5} \mathrm{~nm}$, and are therefore confident about the calibration at the level of $10^{-4} \mathrm{~nm}$. Given the fact that the calibration procedure is repeated at different grating positions, non-linearities in the detector response of the individual pixels (or pixel columns, in the usual binning mode 


\section{J. R. Crespo López-Urrutia et al. / Physics with highly charged...}

applied) and the pixel geometry (for a CCD camera a very small figure) become negligible. The effect from deviations of the line profile from an ideal Gauss has been studied by varying the intervals around the line centre for the fitting procedure in order to take into account or neglect varying amounts of background, scattered light and so on which can affect the line wings. Usually such tests led to centroid shifts of 0.004 pixels on average, or $3.5 \cdot 10^{-5} \mathrm{~nm}$, although in some cases the shift can be as big as $1.5 \cdot 10^{-4} \mathrm{~nm}$ under very unrealistic choices of the fitting interval. This systematic effect could cause an estimated error of $10^{-4} \mathrm{~nm}$ under conservative assumptions. Another possible source of systematic deviations is the location of the spectral lamp in the optical setup. We have performed stepwise changes in this location, resulting in shifts of the order of $10^{-4} \mathrm{~nm}$, consistent with the statistical quality of the data. Temperature drifts as possible sources of systematic errors are ruled out by our calibration procedure. The time analysis of the data shows only statistical departures from the average wavelength value, and no systematic drifts whatsoever. Possible influences from the Zeeman or Stark effect on the wavelength can be ruled out by simple estimates.

Table 1. Experimental wavelengths of some forbidden transitions (preliminary)

\begin{tabular}{|c|c|c|c|}
\hline Ion & Transition & $\begin{array}{c}\text { Wavelength } \\
(\mathrm{nm})\end{array}$ & $\begin{array}{c}\text { Exp. Error } \\
(\mathrm{nm})\end{array}$ \\
\hline $\mathrm{Ar}^{9+}(\mathrm{ArX})$ & $2 \mathrm{~s}^{2} 2 \mathrm{p}^{5}{ }^{2} \mathrm{P}_{3 / 2}{ }^{2} \mathrm{P}_{1 / 2}$ & 553.3260 & 0.0006 \\
\hline $\mathrm{Ar}^{13+}(\mathrm{ArXIV})$ & $2 \mathrm{~s}^{2} 2 \mathrm{p}^{2} \mathrm{P}_{3 / 2}{ }^{2} \mathrm{P}_{1 / 2}$ & 441.2567 & 0.0004 \\
\hline $\mathrm{Ar}^{14+}(\mathrm{ArXV})$ & $2 \mathrm{~s}^{2} \mathrm{P}_{3^{-}}{ }^{3} \mathrm{P}_{2}$ & 594.3884 & 0.0006 \\
\hline
\end{tabular}

Measurements for different highly charged argon ions were carried out in order to obtain a systematic series for systems with 4, 5,8 and 9 electrons. Also some interesting krypton forbidden transitions were observed. All these lines are of potential application for diagnostic purposes (e. g. Doppler shift of solar flares). These (preliminary) results are shown in Table 1. The experimental error is mainly determined by the statistics of the measured lines in comparison with the calibration lines, and finally by the quality of the calibration lines. With a total error of roughly $1 \mathrm{ppm}$, these results belong to the few at this level of accuracy for highly charged ions. We are not aware of any other data with an error bar of less than $7 \mathrm{ppm}$ for visible radiation from highly charged ions. In the x-ray region, very few measurements have an accuracy of better than $20 \mathrm{ppm}$ (again for highly charged ions). Therefore, the present data are well suited to test relativistic 
atomic physics structure codes for few-electron systems with an unprecedented precision.

Another different type of measurement was carried by using a wide open slit. In this way, a multi-wavelength imaging of the spatial distribution of the ions in the trap was obtained. Together with the Doppler-width data obtained in the first set of measurements, detailed information about the thermal velocity and distribution of the ions are obtained, which are necessary to derive cross sections for excitation and recombination processes in the trap.

\section{Dielectronic recombination}

Dielectronic recombination studies have been carried out with highly charged argon ions by using an electron beam of variable energy and recording the photons emitted during the radiative stabilization of the doubly excited states populated by the DR process. The photon energy is measured with a high-purity

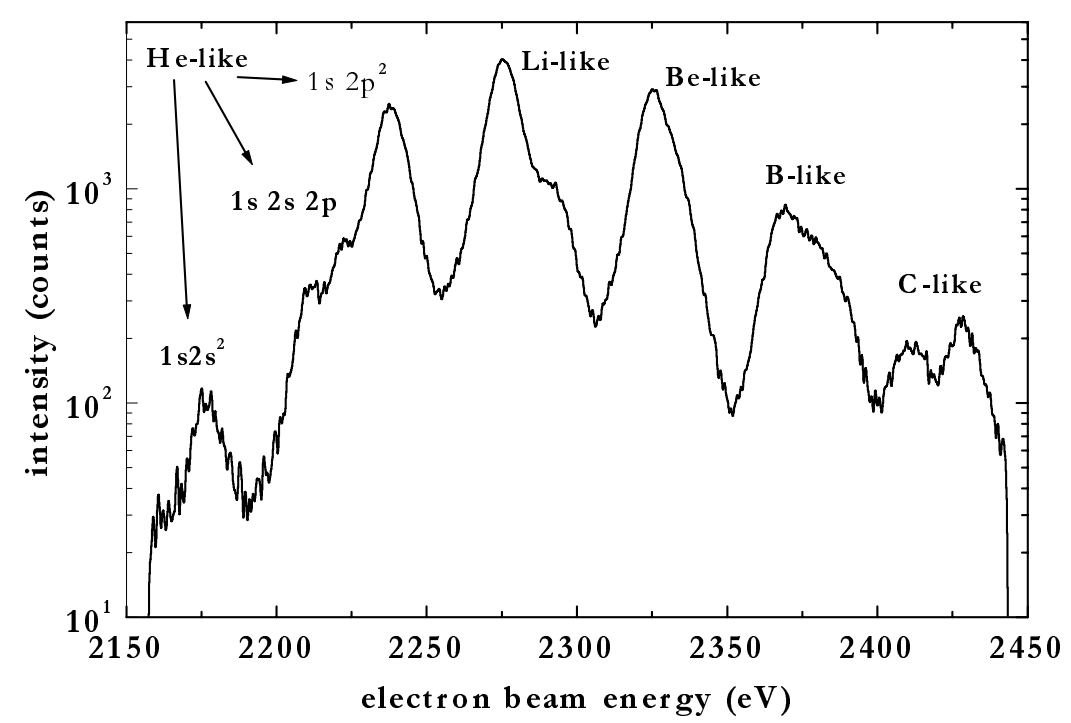

Figure 1. Photon yield of the 2-1 transitions after excitation of the KLL dielectronic resonances of highly-charged Ar ions as a funtion of electron beam energy.

germanium detector with a resolution of roughly $150 \mathrm{eV}$. The beam energy was scanned from 1.5 to $4.5 \mathrm{keV}$. By using very low beam currents, the energy spread of the electron beam, which is mainly caused by its own space charge, was re- 
J. R. Crespo López-Urrutia et al. / Physics with highly charged...

duced to levels as low as $8 \mathrm{eV} \mathrm{FWHM.} \mathrm{This} \mathrm{allowed} \mathrm{us} \mathrm{to} \mathrm{resolve} \mathrm{the} \mathrm{structure}$ of the KLL resonance and to separate the contributions from $\mathrm{He}, \mathrm{Li}, \mathrm{Be}$ and $\mathrm{B}$ like satellites (see Fig.1). The absolute resonance energy was determined by varying the electron beam current from $3 \mathrm{~mA}$ to $50 \mathrm{~mA}$ and extrapolating the space charge shift to zero current, with an estimated absolute error of about 0.5 $\mathrm{eV}$. In the preliminary analysis of the data two important features appear clearly resolved: resonantly excited two-electron-one-photon transitions and the twophoton deexcitation continuum.

\section{Ion-Atom collisions}

Extracted ions $\left(\mathrm{Ne}^{8+}, \mathrm{Ne}^{7+}\right)$ were transported to a COLTRIMS spectrometer to study state-selective charge exchange processes in ion-atom collisions with an excellent energy resolution of $0.7 \mathrm{eV}$ []. In future kinematically complete measurements of multi-electron transfer reactions accompanied by subsequent autoionization of effectively populated highly excited projectile states can be anticipated yielding detailed information about the many electron dynamics in slow highly charged ion-atom collisions. At the new location of FreEBIT at the MaxPlanck-Institut für Kernphysik in Heidelberg, where the facility has been transferred in July 2001, several beamlines are under construction to use the exctracted highly charged ions in addition for surface studies and for high precision experiments after retrapping specific ions in an external ion trap. A laser spectroscopy laboratory is under construction which will allow high resolution hyperfine structure measurements of hydrogen-like ions.

Acknowledgments: We thank the Deutsche Forschunggemeinschaft for financial support under contract No. UL 166/2-1 and from the Leibniz Award 1999.

\section{References}

[1] J. R. Crespo López-Urrutia et al., Phys. Scr. T80(1999)502 ; J. R. Crespo López-Urrutia et al., Hyp. Int. 127 (2000) 497-501; J. R. Crespo López-Urrutia et al., Phys. Scr. T92 (2001) 110-112

[2] J. Ullrich et al., J. Phys. B 30 (1997)2917 (topical review).

[3] D. Fischer et al., APAC2001 proceedings 